\title{
Erratum: Description of a stochastic system by a nonadapted stochastic process [Phys. Rev. E 104, 014139 (2021)]
}

\author{
Piero Olla $(\mathbb{D}$
}

(Received 15 November 2021; published 29 November 2021)

DOI: 10.1103/PhysRevE.104.059901

Equations (57) and (58) in the original paper do not follow from Eqs. (55) and (56). Linearity is not sufficient for $\langle\mathbf{E} \mid \mathcal{B}\rangle$ to be expressed as a sum of contributions proportional to $\left\langle\mathbf{E} \mid \mathcal{B}_{1,2}\right\rangle$. To avoid making the test on linear systems in Sec. IV of the paper ineffectual, an alternative derivation of the equations is required. We provide such derivation below, starting from the Brownian bridge expression for the conditional PDF,

$$
\rho(\mathbf{E} \mid \mathcal{B}) \sim \frac{\rho\left(\mathbf{E} \mid \mathcal{B}_{1}\right) \rho\left(\mathbf{E} \mid \mathcal{B}_{2}\right)}{\rho(\mathbf{E})},
$$

where $\rho(\mathbf{E}) \sim \exp \left[-|\mathbf{E}|^{2} /(2 \mathcal{E})\right]$ is the equilibrium PDF, and we are hereafter neglecting normalization factors. The conditional PDF's $\rho\left(\mathbf{E} \mid \mathcal{B}_{1,2}\right)$ can be expressed in terms of correlations $C_{i j}(t)=\left\langle E_{i}(0) E_{j}(t)\right\rangle$ and propagators $G_{i j}(t)=\delta E_{i}(t) / \delta \xi_{j}(0)$ as follows:

$$
\begin{aligned}
& \rho\left(\mathbf{E} \mid \mathcal{B}_{1}\right) \sim \exp \left[-\frac{R_{i j}^{i}}{2}\left(E_{i}-G_{i 1}^{i} E_{1}^{i}\right)\left(E_{j}-G_{j 1}^{i} E_{1}^{i}\right)\right], \\
& \rho\left(\mathbf{E} \mid \mathcal{B}_{2}\right) \sim \exp \left[-\frac{R_{i j}^{f}}{2}\left(E_{i}-G_{i 2}^{f} E_{2}^{f}\right)\left(E_{j}-G_{j 2}^{f} E_{2}^{f}\right)\right],
\end{aligned}
$$

where to $O(g)$,

$$
\begin{array}{lll}
R_{11}^{i}=1 / C_{11}^{i}, & R_{22}^{i}=1 / \mathcal{E}, & R_{12}^{i}=-g C_{12}^{i(1)} / C_{11}^{i(0)}, \\
R_{11}^{f}=1 / \mathcal{E}, & R_{22}^{f}=1 / C_{11}^{f}, & R_{12}^{f}-g C_{12}^{f(1)} / C_{11}^{f(0)},
\end{array}
$$

where $R_{i j}=R_{j i}$, for the system under consideration $C_{11}=C_{22}$, and where superscripts $i$ and $f$ on $C$ and $G$ indicate dependence on time differences $t-t_{i}$ and $t_{f}-t$, respectively.

Because of linearity of the dynamics, the conditional moments $\langle\mathbf{E} \mid \mathcal{B}\rangle$ depend linearly on $E_{1}^{i}$ and $E_{2}^{f}$. We can thus linearize $\rho\left(\mathbf{E} \mid \mathcal{B}_{1,2}\right)$ with respect to $E_{1}^{i}$ and $E_{2}^{f}$ :

$$
\begin{gathered}
\rho\left(\mathbf{E} \mid \mathcal{B}_{1}\right) \sim \exp \left[-\frac{1}{2}\left(\frac{E_{1}^{2}}{C_{11}^{i}}+\frac{E_{2}^{2}}{\mathcal{E}}\right)+g \frac{C_{12}^{i(1)}}{C_{11}^{i(0)}} E_{1} E_{2}\right]\left\{1+\left[\frac{G_{11}^{i}}{C_{11}^{i}} E_{1}+g\left(G_{12}^{i(1)}-\frac{C_{12}^{i(1)} G_{11}^{i(0)}}{C_{11}^{i(0)}}\right) E_{2}\right] E_{1}^{i}\right\}, \\
\rho\left(\mathbf{E} \mid \mathcal{B}_{2}\right) \sim \exp \left[-\frac{1}{2}\left(\frac{E_{1}^{2}}{\mathcal{E}}+\frac{E_{2}^{2}}{C_{11}^{f}}\right)+g \frac{C_{12}^{f(1)}}{C_{11}^{f(0)}} E_{1} E_{2}\right]\left\{1+\left[\frac{G_{11}^{f}}{C_{11}^{f}} E_{2}+g\left(G_{12}^{f(1)}-\frac{C_{12}^{f(1)} G_{11}^{f(0)}}{C_{11}^{f(0)}}\right) E_{1}\right] E_{2}^{f}\right\} .
\end{gathered}
$$

We substitute Eqs. (2) and (3) into Eq. (1), keeping terms up to $O(g)$, and obtain

$$
\begin{aligned}
\rho(\mathbf{E} \mid \mathcal{B}) \sim & \left\{1+\left[\frac{G_{11}^{i}}{C_{11}^{i}} E_{1}+g\left(G_{12}^{i(1)}-\frac{C_{12}^{i(1)} G_{11}^{i(0)}}{C_{11}^{i(0)}}\right) E_{2}\right] E_{1}^{i}+\left[\frac{G_{11}^{f}}{C_{11}^{f}} E_{2}+g\left(G_{12}^{f(1)}-\frac{C_{12}^{f(1)} G_{11}^{f(0)}}{C_{11}^{f(0)}}\right) E_{1}\right] E_{2}^{f}\right\} \\
& \times\left[1+g\left(\frac{C_{12}^{i(1)}}{C_{11}^{i(0)}}+\frac{C_{12}^{f(1)}}{C_{11}^{f(0)}}\right) E_{1} E_{2}\right] \exp \left[-\frac{1}{2}\left(\frac{E_{1}^{2}}{C_{11}^{i}}+\frac{E_{2}^{2}}{C_{11}^{f}}\right)\right] .
\end{aligned}
$$

Expressing $\langle\mathbf{E} \mid \mathcal{B}\rangle$ as a superposition of contributions by $\left\langle\mathbf{E} \mid \mathcal{B}_{1}\right\rangle$ and $\left\langle\mathbf{E} \mid \mathcal{B}_{2}\right\rangle$, as suggested in the original paper, would miss the contribution from the third line in Eq. (4).

From Eq. (4) we get the expression for the conditional moments, correct to $O(g)$,

$$
\begin{aligned}
& \left\langle E_{1} \mid \mathcal{B}\right\rangle=G_{11}^{i} E_{1}^{i}+(g / \mathcal{E})\left(G_{12}^{f(1)} C_{11}^{i(0)}+C_{12}^{i(1)} G_{11}^{f(0)}\right) E_{2}^{f}, \\
& \left\langle E_{2} \mid \mathcal{B}\right\rangle=G_{11}^{f} E_{2}^{f}+(g / \mathcal{E})\left(G_{12}^{i(1)} C_{11}^{f(0)}+C_{12}^{f(1)} G_{11}^{i(0)}\right) E_{1}^{i} .
\end{aligned}
$$


Expressions for the propagators $G_{i j} \simeq G_{i j}^{(0)}+g G_{i j}^{(1)}$ and the correlation functions $C_{i j} \simeq C_{i j}^{(0)}+g C_{i j}^{(1)}$ can be obtained directly from Eqs. (4), (49), and (50) in the original paper. We get

$$
\begin{aligned}
& C_{11}^{(0)}(t)=\mathcal{E}\left(1-e^{-2|t|}\right), \quad C_{12}^{(1)}(t)=-\mathcal{E}|t| e^{-2|t|}, \\
& G_{11}(t) \simeq(1-g t) \theta(t) e^{-t}, \quad G_{12}^{(1)}(t)=t \theta(t) e^{-t},
\end{aligned}
$$

which, by substituting into Eqs. (5) and (6) above, yield Eqs. (57) and (58) in the original paper.

We note the statement in the caption to Fig. 2 in the original paper, that the green curves in the figure are obtained using Eqs. (55) and (56). Actually, those curves were obtained numerically from the Brownian bridge expression for $\rho(\mathbf{E} \mid \mathcal{B})[$ Eq. (1) in the present erratum]. Thus, although the statement in the caption is incorrect, the figure does not need to be amended.

There are additional typographical errors in the original paper, which must be corrected:

(1) In Eq. (5), $F_{j}^{(1)}$ must be replaced by $F_{j}$.

(2) In Eq. (22), $M_{1}^{(1)}(n+1)$ must be replaced by $M_{2}^{(1)}(n+1)$.

(3) In Eq. (52), $\dot{E}_{2}-E_{1}+\cdots$ must be replaced by $\dot{E}_{2}-E_{2}+\cdots$. 